\title{
Distributed Iteratively Quantized Kalman Filtering for Wireless Sensor Networks
}

\author{
Eric J. Msechu, Alejandro Ribeiro, Stergios I. Roumeliotis and Georgios B. Giannakis
}

\begin{abstract}
We consider state estimation of a dynamic process using a wireless sensor network (WSN). Due to bandwidth constraints, analog amplitude observations are quantized to $m$-bit messages, based on which the state is estimated. We introduce a novel iterative quantization approach such that at time $n$, the $i$-th bit is iteratively formed using the sign of the difference between the $n$-th observation and its estimate based on past observations (up to time $n-1$ ) along with previous bits (up to $i-1)$ of the current observation. Analysis and simulations confirm that KF-like tracking based on only 2 to 4 bits of iteratively quantized data communicated among sensors exhibits meansquare error (MSE) performance identical to a KF based on analog-amplitude data.
\end{abstract}

Technical Areas: C.2 Wireless sensor networks, E.6 Estimation and detection.

\section{INTRODUCTION}

Consider an ad-hoc wireless sensor network (WSN) deployed to track a Markov stochastic process. Each sensor in the network takes observations given as linear transformation of the common state. The sensors then transmit observations to each other in order to build a state estimate. If observations were available at a common location minimum mean squared error (MMSE) estimates could be obtained using a Kalman filter (KF). However, since observations are distributed in space and there is a constraint on communication bandwidth, the observations have to be quantized before transmission. Thus, the original estimation problem is transformed into distributed state estimation using quantized observations.

State estimation using quantized observations is a non-linear estimation problem. As such it requires the use of computationally expensive tools, e.g., unscented (U)KFs, or particle filters [3]. Quite surprisingly, for the case where quantized observations are defined as the sign of the innovation (SoI) sequence it is possible to derive a filter with complexity and performance very close to the clairvoyant KF based on the original observations [6]. However promising, the approach of [6] is limited to a particular binary quantizer.

Here, we consider quantization to an arbitrary number of bits $m$. We propose an iterated quantizer whereby at time $n$ the $i$-th bit is iteratively formed using the sign of the difference between the $n$-th observation and its estimate based on past observations (up to time $n-1$ ) along with previous bits (up to $i-1$ ) of the current observation. Analysis and simulations confirm that KF-like tracking based on $m$ bits of iteratively quantized data communicated among sensors exhibits mean-square error (MSE) performance virtually coincident to that of a KF based on analogamplitude data.

\section{MODELS AND PROBLEM STATEMENT}

Consider an ad-hoc WSN whose $K$ sensor nodes $\left\{\mathcal{S}_{k}\right\}_{k=1}^{K}$ estimate a multivariate random process $\mathbf{x}(n) \in \mathbb{R}^{p}$. State evolution and observations follow the linear model

$$
\begin{aligned}
\mathbf{x}(n) & =\mathbf{A}(n) \mathbf{x}(n-1)+\mathbf{u}(n) \\
y_{k}(n) & =\mathbf{h}_{k}^{T}(n) \mathbf{x}(n)+v_{k}(n),
\end{aligned}
$$

where the noise processes $\{\mathbf{u}(n)\},\left\{v_{k}(n)\right\}$ are zero-mean white Gaussian with autocorrelation $\mathrm{E}\left\{\mathbf{u}\left(n_{1}\right), \mathbf{u}\left(n_{2}\right)\right\}=\mathbf{C}_{u}(n) \delta\left(n_{1}-\right.$ $\left.n_{2}\right)$ and $\mathrm{E}\left\{v_{k}\left(n_{1}\right) v_{l}\left(n_{2}\right)\right\}=c_{v}(t) \delta_{c}\left(n_{1}-n_{2}\right) \delta(k-l)$. It is further assumed that $\mathbf{u}(n)$ is independent of $v_{k}(n)$.

For convenience we augment the state vector $\mathbf{x}(n)$ to include the observation noise $v(n)$, see e.g. [4, Ch. 4.11]. We thus define the augmented state sequence $\breve{\mathbf{x}}(n)=\left[\mathbf{x}^{T}(n), v(n)\right]^{T}$. Further defining the augmented driving input $\breve{\mathbf{u}}(n):=\left[\mathbf{u}^{T}(n), v(n)\right]^{T}$ we can write

$$
\begin{aligned}
\breve{\mathbf{x}}(n) & =\breve{\mathbf{A}}(n) \breve{\mathbf{x}}(n-1)+\breve{\mathbf{u}}(n) \\
\breve{y}(n) & =\breve{\mathbf{h}}^{T}(n) \breve{\mathbf{x}}(n) .
\end{aligned}
$$

where we defined $\breve{\mathbf{A}}(n)=\left(\begin{array}{cc}\mathbf{A}(n) & \mathbf{0} \\ \mathbf{0}^{T} & 0\end{array}\right)$ and $\breve{\mathbf{h}}(n)=$ $\left[\mathbf{h}^{T}(n), 1\right]^{T}$ so that the model in (3)-(4) is equivalent to the model in (1)-(2). Further note that $\breve{y}(n)=y(n)$ and that the correlation matrix $\mathbf{C}_{\breve{\mathbf{u}}}(n) \in \mathbb{R}^{(p+1) \times(p+1)}$ is block-diagonal with $\left[\mathbf{C}_{\breve{\mathbf{u}}}(n)\right]_{1: p, 1: p}=\mathbf{C}_{\mathbf{u}}(n)$ and $\left[\mathbf{C}_{\breve{\mathbf{u}}}(n)\right]_{p+1, p+1}=c_{v}(n)$. When estimating the augmented state $\breve{\mathbf{x}}(n)$ we estimate the original state $\mathbf{x}(n)$ and the observation noise $v(n)$. This formulation will simplify the derivation of our filter.

Assuming that $\mathbf{A}(n), \mathbf{C}_{u}(n), \mathbf{h}_{k}(n)$ and $c_{v_{k}}(n)$ are available $\forall n, k$, the goal of the WSN is for each sensor $\mathcal{S}_{k}$ to form an estimate of $\mathbf{x}(n)$. This necessitates each sensor $\mathcal{S}_{l}$ to communicate $y_{l}(n)$ to the remaining sensors $\left\{\mathcal{S}_{k}\right\}_{k=1, k \neq l}^{K}$ over the shared bandwidth-constrained wireless channel. We will assume the transmission of a single packet of $m$ bits per time slot $n$, leading to a one-to-one correspondence between time $n$ and sensor index $k$ and allowing us to drop the sensor index $k$ in (2).

To construct this $m$-bit message we define a quantization function $\mathbf{q}_{n}$ to map the observation $y(n)$ to the message $\mathbf{b}(n)$, i.e., $\mathbf{b}(n):=\mathbf{q}_{n}(y(n))$. Given current and past messages $\mathbf{b}_{1: n}:=\left[\mathbf{b}^{T}(1), \ldots, \mathbf{b}^{T}(n)\right]^{T}$ the objective is to compute MMSE estimates of $\breve{\mathbf{x}}(n)$ that we denote as $\hat{\mathbf{x}}\left(n \mid \mathbf{b}_{1: n}\right)$. As is well known, see e.g., [4, chapter 5], $\hat{\mathbf{x}}\left(n \mid \mathbf{b}_{1: n}\right)$ is given by the conditional expectation

$$
\hat{\widehat{\mathbf{x}}}\left(n \mid \mathbf{b}_{1: n}\right):=\mathrm{E}\left\{\breve{\mathbf{x}}(n) \mid \mathbf{b}_{1: n}\right\}=\int_{\mathbb{R}^{p}} \breve{\mathbf{x}}(n) p\left[\breve{\mathbf{x}}(n) \mid \mathbf{b}_{1: n}\right] d \breve{\mathbf{x}}(n)
$$

where $p\left[\breve{\mathbf{x}}(n) \mid \mathbf{b}_{1: n}\right]$ denotes the conditional distribution of $\breve{\mathbf{x}}(n)$ given messages $\mathbf{b}_{1: n}$. For future reference define the error 
covariance matrix (ECM) of the estimator as $\breve{\mathbf{M}}\left(n \mid \mathbf{b}_{1: n}\right):=$ $\mathrm{E}\left\{\left[\hat{\tilde{\mathbf{x}}}\left(n \mid \mathbf{b}_{1: n}\right)-\breve{\mathbf{x}}(n)\right]\left[\hat{\mathbf{x}}\left(n \mid \mathbf{b}_{1: n}\right)-\breve{\mathbf{x}}(n)\right]^{T}\right\}$.

Our goal in this paper is to propose an iterative quantization rule so that the estimator in (5) can be approximated using a prediction-correction form similar to the KF recursion [4, chapter 5]. We will furthermore discuss preliminary results suggesting that our filter performs surprisingly close to a KF relying on unquantized observations, even when the number of bits $m$ is very small.

\section{DistRIBUted ITERATED MULTI-BIT KALMAN FILTER}

At time index $\mathrm{n}$, an $m$-bit binary message $\mathbf{b}^{(1: m)}(n):=$ $\left[b^{(1)}(n), \ldots, b^{(m)}(n)\right]^{T}$ is defined, where the $i$-th bit $b^{(i)}(n)$ is a function of the previous messages $\mathbf{b}_{1: n-1}:=\left[\mathbf{b}^{T}(1), \ldots, \mathbf{b}(n-\right.$ 1) $]^{T}$ and previous bits of the current message $\mathbf{b}^{(1: i-1)}(n):=$ $\left[b^{(1)}(n), \ldots, b^{(i-1)}(n)\right]^{T}$. Specifically define the MMSE observation prediction

$$
\hat{y}^{(i)}\left(n \mid \mathbf{b}_{1: n-1}\right):=\mathrm{E}\left\{y(n) \mid \mathbf{b}_{1: n-1}, \mathbf{b}^{(1: i-1)}(n)\right\}
$$

that estimates $y(n)$ using past messages $\mathbf{b}_{1: n-1}$ and the first $i-1$ bits of the current message $\mathbf{b}^{(1: i-1)}(n)$. The $i$-th bit $b^{(i)}(n)$ of the current message is then defined as the difference between the actual observation and the observation prediction, i.e.,

$$
b^{(i)}(n):=\operatorname{sign}\left[y(n)-\hat{y}^{(i)}\left(n \mid \mathbf{b}_{1: n-1}\right)\right]
$$

where we adopt the convention that for $i=1, \hat{y}^{(1)}\left(n \mid \mathbf{b}_{1: n-1}\right):=$ $\hat{y}\left(n \mid \mathbf{b}_{1: n-1}\right)$.

For messages defined as in (6)-(7) computing the MMSE estimates in (5) is challenging due to the non-linear nature of the relation between the state $\breve{\mathbf{x}}(n)$ and the quantized messages $\mathbf{b}(n)$. Quite surprisingly, it is possible to compute approximate MMSE estimates with a low-complexity recursion, as we detail in the next proposition.

Proposition 1 Consider the augmented state space model in (3) and (4) and binary observations as in (7) with $\hat{y}^{(i)}\left(n \mid \mathbf{b}_{1: n-1}\right):=$ $\breve{\mathbf{h}}^{T}(n) \hat{\breve{\mathbf{x}}}^{(i)}\left(n \mid \mathbf{b}_{1: n-1}\right) . \quad$ If $\quad p\left[\breve{\mathbf{x}}(n) \mid \mathbf{b}_{1: n-1}, \mathbf{b}^{(1: i)}(n)\right]=$ $\mathcal{N}\left[\breve{\mathbf{x}}(n) ; \hat{\mathbf{x}}^{(i)}\left(n \mid \mathbf{b}_{1: n-1}\right), \breve{\mathbf{M}}^{(i)}\left(n \mid \mathbf{b}_{1: n-1}\right)\right]$ for $i \in[1, m]$, then the MMSE estimator of $\breve{\mathbf{x}}(n)$ based on $\mathbf{b}_{1: n-1}$ and $\mathbf{b}^{(1: m)}(n)$ is obtained from the following recursion:

[S1] Define the predicted estimate $\hat{\tilde{\mathbf{x}}}\left(n \mid \mathbf{b}_{1: n-1}\right) \quad:=$ $\mathrm{E}\left\{\breve{\mathbf{x}}(n) \mid \mathbf{b}_{1: n-1}\right\} \quad$ and let $\breve{\mathbf{M}}\left(n \mid \mathbf{b}_{1: n-1}\right) \quad:=$ $\mathrm{E}\left\{\left[\hat{\mathbf{x}}\left(n \mid \mathbf{b}_{1: n-1}\right)-\breve{\mathbf{x}}(n)\right]\left[\hat{\mathbf{x}}\left(n \mid \mathbf{b}_{1: n-1}\right)-\breve{\mathbf{x}}(n)\right]^{T}\right\}$ denote the corresponding ECM. Given the previous estimate $\hat{\mathbf{x}}\left(n-1 \mid \mathbf{b}_{1: n-1}\right)$ and its ECM $\breve{M}\left(n-1 \mid \mathbf{b}_{1: n-1}\right)$,

$$
\begin{aligned}
\hat{\hat{\mathbf{x}}}\left(n \mid \mathbf{b}_{1: n-1}\right) & =\breve{\mathbf{A}}(n) \hat{\hat{\mathbf{x}}}\left(n-1 \mid \mathbf{b}_{1: n-1}\right) \\
\breve{\mathbf{M}}\left(n \mid \mathbf{b}_{1: n-1}\right) & =\breve{\mathbf{A}}(n) \breve{\mathbf{M}}\left(n-1 \mid \mathbf{b}_{1: n-1}\right) \breve{\mathbf{A}}^{T}(n)+\mathbf{C}_{\breve{\mathbf{u}}}(n)
\end{aligned}
$$

[S2] Assuming that $\quad p\left[\breve{\mathbf{x}}(n) \mid \mathbf{b}_{1: n-1}, \mathbf{b}^{(1: i)}(n)\right]$ $\mathcal{N}\left[\breve{\mathbf{x}}(n) ; \hat{\mathbf{x}}^{(i)}\left(n \mid \mathbf{b}_{1: n-1}\right), \breve{\mathbf{M}}^{(i)}\left(n \mid \mathbf{b}_{1: n-1}\right)\right] \quad$ the MMSE estimate $\hat{\mathbf{x}}^{(i)}\left(n \mid \mathbf{b}_{1: n}\right):=\mathrm{E}\left\{\breve{\mathbf{x}}(n) \mid \mathbf{b}_{1: n}, \mathbf{b}^{(1: i)}\right\}$ and the corresponding ECM $\mathbf{M}^{(i)}\left(n \mid \mathbf{b}_{1: n}\right)$ are obtained by iterative application of

$$
\begin{gathered}
\hat{\mathbf{x}}^{(i+1)}\left(n \mid \mathbf{b}_{1: n-1}\right)=\hat{\mathbf{x}}^{(i)}\left(n \mid \mathbf{b}_{1: n-1}\right)+ \\
\sqrt{\frac{2}{\pi}} \frac{\breve{\mathbf{M}}^{(i)}\left(n \mid \mathbf{b}_{1: n-1}\right) \breve{\mathbf{h}}(n)}{\sqrt{\breve{\mathbf{h}}^{T}(n) \breve{M}^{(i)}\left(n \mid \mathbf{b}_{1: n-1}\right) \breve{\mathbf{h}}(n)}} b^{(i)}(n) \\
\breve{\mathbf{M}}^{(i+1)}\left(n \mid \mathbf{b}_{1: n-1}\right)=\breve{\mathbf{M}}^{(i)}\left(n \mid \mathbf{b}_{1: n-1}\right)- \\
\frac{2}{\pi} \frac{\breve{\mathbf{M}}^{(i)}\left(n \mid \mathbf{b}_{1: n-1}\right) \breve{\mathbf{h}}(n) \breve{\mathbf{h}}^{T}(n) \breve{\mathbf{M}}\left(\breve{M}^{(i)}\left(n \mid \mathbf{b}_{1: n-1}\right)\right.}{\breve{\mathbf{h}}^{T}(n) \breve{\mathbf{M}}^{(i)}\left(n \mid \mathbf{b}_{1: n-1}\right) \breve{\mathbf{h}}(n)}
\end{gathered}
$$

where we adopted the convention $\hat{\mathbf{x}}^{(1)}\left(n \mid \mathbf{b}_{1: n-1}\right):=$ $\hat{\mathbf{x}}\left(n \mid \mathbf{b}_{1: n-1}\right)$ and $\breve{\mathbf{M}}^{(1)}\left(n \mid \mathbf{b}_{1: n-1}\right):=\mathbf{M}\left(n \mid \mathbf{b}_{1: n-1}\right)$. Eqs. (10) and (11) are repeated for $i \in[1, m]$.

The MMSE estimate of $\hat{\tilde{\mathbf{x}}}(n)$ given $\mathbf{b}_{1: n}$ is given by $\hat{\tilde{\mathbf{x}}}\left(n \mid \mathbf{b}_{1: n}\right):=$ $\hat{\mathbf{x}}^{(m+1)}\left(n \mid \mathbf{b}_{1: n-1}\right)$. The corresponding ECM is $\mathbf{M}\left(n \mid \mathbf{b}_{1: n}\right):=$ $\breve{M}^{(m+1)}\left(n \mid \mathbf{b}_{1: n-1}\right)$.

The similarity of the algorithm in Proposition 1 and the KF based on analog amplitude observations is remarkable. In particular, the ECM updates in (11) are identical to the ECM updates of the KF except for the (small) factor $\pi / 2$. This suggests that the MSE penalty due to quantization is usually small. This issue is further explored in the following corollaries.

Corollary 1 Consider the algorithm in Proposition 1 and define the ECM reduction after $i \in[1, M]$ iterations as $\Delta \breve{\mathbf{M}}_{i}(n):=\breve{\mathbf{M}}^{(1)}\left(n \mid \mathbf{b}_{1: n-1}\right)-\breve{\mathbf{M}}^{(i+1)}\left(n \mid \mathbf{b}_{1: n-1}\right)$, where $\breve{\mathbf{M}}^{(1)}\left(n \mid \mathbf{b}_{1: n-1}\right):=\mathbf{M}\left(n \mid \mathbf{b}_{1: n-1}\right)$. After $m$ iterations the covariance reduction is:

$$
\Delta \breve{\mathbf{M}}_{m}(n)=c_{m} \frac{\breve{\mathbf{M}}^{(1)}\left(n \mid \mathbf{b}_{1: n-1}\right) \breve{\mathbf{h}}(n) \breve{\mathbf{h}}^{T}(n) \breve{\mathbf{M}}^{(1)}\left(n \mid \mathbf{b}_{1: n-1}\right)}{\breve{\mathbf{h}}^{T}(n) \breve{\mathbf{M}}^{(1)}\left(n \mid \mathbf{b}_{1: n-1}\right) \breve{\mathbf{h}}(n)}
$$

with $c_{m}:=\left[1-\left(1-\frac{2}{\pi}\right)^{m}\right]$.

For the clairvoyant KF based on unquantized observations the ECM reduction is as in (12) with $c_{m}=1$. Consequently, Corollary 1 tells us that the covariance reduction achieved by quantizing to $m$ bits is $c_{m}$ times smaller than the one achieved with analog observations. Values of $c_{m}$ are shown in Table I. With 4 bits of quantization, the value of $c_{m}$ is only $2 \%$ less than the value for the clairvoyant $\mathrm{KF}\left(c_{m}=1\right)$.

We further see that as $m \rightarrow \infty, c_{m} \rightarrow 1$. Thus, for infinite number of quantization bits, we recover the clairvoyant KF. This important consistency result is summarized in the following corollary.

Corollary 2 For $m \rightarrow \infty$ the ECM of the filter in Proposition 1 $\overrightarrow{\mathbf{M}}\left(n \mid \mathbf{b}_{1: n}\right)=\mathbf{M}^{(m+1)}\left(n \mid \mathbf{b}_{1: n-1}\right)=\mathbf{M}\left(n \mid \mathbf{b}_{1: n-1}\right)-\Delta \mathbf{M}_{m}(n)$ becomes identical to that of the clairvoyant $\operatorname{KF}_{\mathbf{M}}^{K}\left(n \mid \mathbf{b}_{1: n}\right)=$ $\breve{\mathbf{M}}\left(n \mid \mathbf{b}_{1: n-1}\right)-\Delta \breve{\mathbf{M}}^{K}(n)$, i.e.,

$$
\lim _{m \rightarrow \infty} \breve{\mathbf{M}}^{(m+1)}\left(n \mid \mathbf{b}_{1: n-1}\right)=\breve{\mathbf{M}}^{\mathrm{K}}\left(n \mid \mathbf{b}_{1: n}\right) .
$$

We remark that Corollary 1 quantifies the per time step ECM reduction for the proposed filter. Because of error accumulation we could expect that the MSE of our filter given as $\operatorname{tr}\left\{\mathbf{M}\left(n \mid \mathbf{b}_{1: n}\right)\right\}$ and the MSE of the clairvoyant $\mathrm{KF} \operatorname{tr}\left\{\mathbf{M}\left(n \mid \mathbf{y}_{1: n}\right)\right\}$ become significantly different as time progresses. Quite interestingly, the 
TABLE I

PERFORMANCE PENALTY FOR DIFFERENT NUMBER OF QUANTIZATION BITS.

\begin{tabular}{|l|l|l|l|l|}
\hline bits, $m$ & 1 & 2 & 3 & 4 \\
\hline$c_{m} * 100 \%$ & $63.66=2 / \pi * 100$ & 86.80 & 95.20 & 98.26 \\
\hline
\end{tabular}

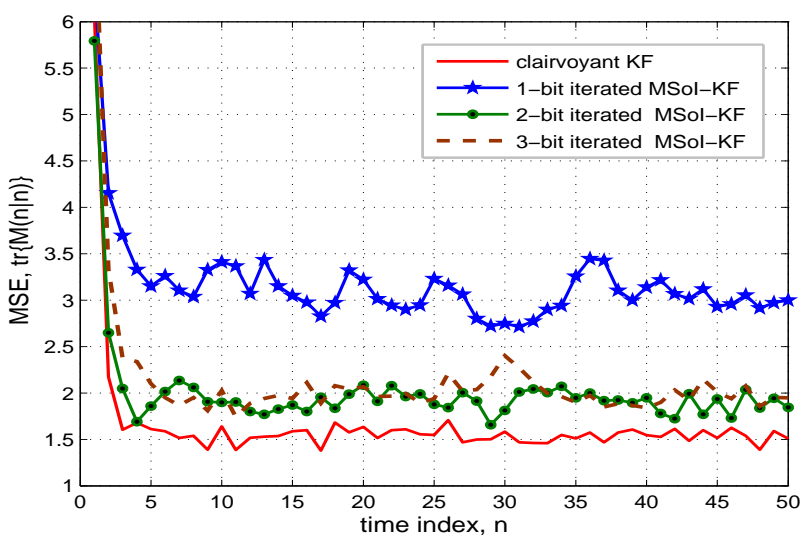

Fig. 1. Empirically computed estimator $\operatorname{MSE} \operatorname{tr}\left\{\mathbf{M}\left(n \mid \mathbf{b}_{1: n}\right)\right\}$ for 1-3 bits of quantization. MSE for clairvoyant KF is included for comparison.

simulated experiments presented in the next section show that the relative penalties of Table 1 are also valid when comparing the $\operatorname{tr}\left\{\mathbf{M}\left(n \mid \mathbf{b}_{1: n}\right)\right\}$ and $\operatorname{tr}\left\{\mathbf{M}\left(n \mid \mathbf{y}_{1: n}\right)\right\}$ To understand why this happens we have to look at the Riccati equations. This will be done in the final version of the manuscript.

\section{Preliminary Simulations}

For a two dimensional model, Fig. 1 compares the MSEs $\operatorname{tr}\left\{\mathbf{M}\left(n \mid \mathbf{y}_{1: n}\right)\right\}$ and $\operatorname{tr}\left\{\mathbf{M}\left(n \mid \mathbf{b}_{1: n}\right)\right\}$ for $m=1, m=2$ and $m=3$ bits. The plot shows a substantial MSE reduction when going from $m=1$ bit to $m=2$ bits and a small performance gain for larger $m$. With $m=2$ bits the MSE performance is virtually identical to that of the clairvoyant KF. This corroborates the analytical result of Corollary 1 since according to Table I $c_{2}=86.8 \%$ and $c_{3}=95.2 \%$.

In Figs. 2 and 3 we check the consistency of the estimators by comparing the estimated MSE $\operatorname{tr}\left\{\mathbf{M}\left(n \mid \mathbf{b}_{1: n}\right)\right\}$ and the predicted $\operatorname{MSEtr}\left\{\mathbf{M}\left(n \mid \mathbf{b}_{1: n-1}\right)\right\}$ with the corresponding values obtained from the sample covariances. In Fig 2 we see that the analytical and sampled MSEs match well. Fig. 3 shows the consistency tests for the iterated SoI-KF algorithm using the normalized estimation error squared (NEES) tests [1, Ch. 5.4]. Using 200 iterations and $95 \%$ acceptance region we observed only $7 \%$ of 200 time samples were outside the acceptance region.

\section{CONCLUDING REMARKS}

A recursive state estimator based on compressed observation data was considered. Compression is achieved by iterative one step quantization of the innovations to a block of bits. Motivated by the need to find quantifiable trade-offs between estimator performance and number of quantization bits for distributed estimation, it was demonstrated that quantization using 2 to 3 bits improves the performance of the iterative multi-bit KF algorithm to virtually coincide with the optimal state estimator (clairvoyant Kalman Filter) with only minimal increase in computation.

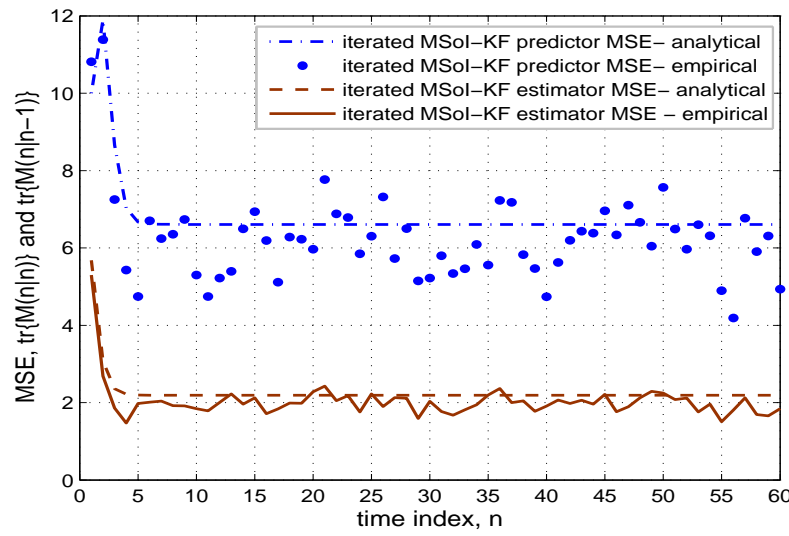

Fig. 2. Consistency test: Empirical MSE (from Monte Carlo data) versus Analytical MSE, for both predictor ECM $\mathbf{M}\left(n \mid \mathbf{b}_{1: n-1}\right)$ and estimator $\operatorname{ECM~} \mathbf{M}\left(n \mid \mathbf{b}_{1: n}\right)$.

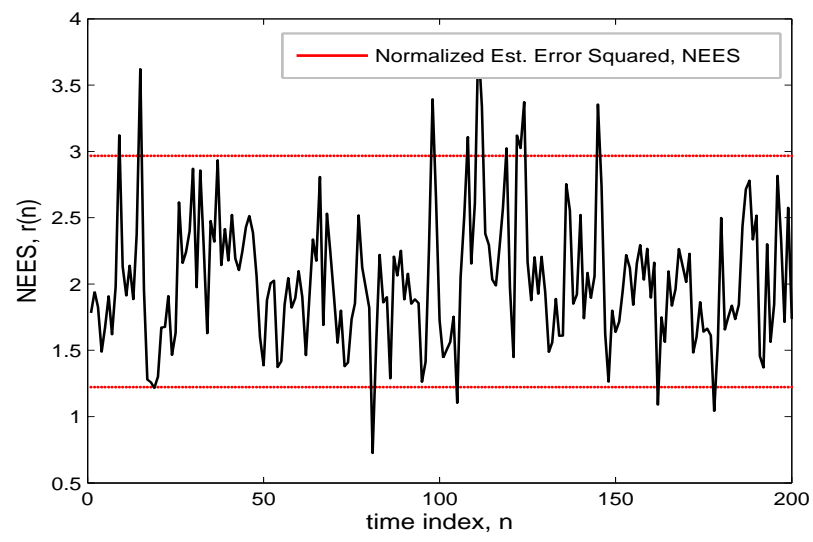

Fig. 3. Consistency test using normalized estimation error squared (NEES), $r(n)=\left[\mathbf{x}(n)-\hat{\mathbf{x}}\left(n \mid \mathbf{b}_{1: n}\right)\right]^{T} \mathbf{M}\left(n \mid \mathbf{b}_{1: n}\right)^{-1}\left[\mathbf{x}(n)-\hat{\mathbf{x}}\left(n \mid \mathbf{b}_{1: n}\right)\right]$ compared with the $95 \%$ probability region for 2 bits quantization.

Preliminary simulations corroborate the analytical results for a tracking example using Gauss-Markov linear state space model.

\section{REFERENCES}

[1] Y. Bar-Shalom and X. Li, Estimation and Tracking: Principles, Techniques, and Software. Artech House, 1993.

[2] R. M. Gray, "Quantization in Task-Driven Sensing and Distributed Processing," in Proc. Int. Conf. Acoustics, Speech, Signal Processing, vol. 5, pp. V-1049- V-1052, Toulouse, France, May 14-19, 2006.

[3] S. Julier and J. Uhlmann, "Unscented filtering and nonlinear estimation," Proceedings of the IEEE, vol. 92, pp. 401-422, March 2004.

[4] P. S. Maybeck, Stochastic Models, Estimation and Control - Vol.1. Academic Press, first ed., 1979.

[5] H. Papadopoulos, G. Wornell, and A. Oppenheim, "Sequential signal encoding from noisy measurements using quantizers with dynamic bias control," IEEE Transactions on Information Theory, vol. 47, pp. 978-1002, 2001.

[6] A. Ribeiro, G. B. Giannakis, and S. I. Roumeliotis, "SOI-KF: Distributed Kalman Filtering With Low-Cost Communications Using the Sign of Innovations," IEEE Transactions on Signal Processing, vol. 54, no. 12, pp. 4782-4795, Dec 2006. 